\title{
Microreactor Cells for High-Throughput X-ray Absorption Spectroscopy
}

\author{
Angela Beesley ${ }^{\mathrm{a}}$, Nikolaos Tsapatsaris ${ }^{\mathrm{a}}$, Norbert Weiher ${ }^{\mathrm{a}}$, Moniek Tromp ${ }^{\mathrm{b}}$, John \\ Evans ${ }^{b}$, Andy Dent ${ }^{\mathrm{c}}$, Ian Harvey ${ }^{\mathrm{d}}$, Sven L. M. Schroeder ${ }^{\mathrm{a} \mathrm{e}^{*}}$ \\ ${ }^{a}$ School of Chemical Engineering and Analytical Science, The University of Manchester,Sackville Street, P.O. Box \\ 88. Manchester, M60 1QD, UK. \\ ${ }^{b}$ School of Chemistry, The University of Southhampton, Highfield, Southampton, SO17 1BJ, UK. \\ ${ }^{c}$ Diamond Light Source Ltd., Diamond House, Chilton, Didcot, Oxfordshire, OX11 ODE, UK. \\ ${ }^{d}$ Synchrotron Radiation Source (SRS), Daresbury Laboratory, Warrington, Cheshire, WA4 4AD, UK \\ ${ }^{e}$ School of Chemistry, The University of Manchester,Sackville Street, P.O. Box 88. Manchester, M60 1QD, UK.
}

\begin{abstract}
High-throughput experimentation has been applied to X-ray Absorption spectroscopy as a novel route for increasing research productivity in the catalysis community. Suitable instrumentation has been developed for the rapid determination of the local structure in the metal component of precursors for supported catalysts. An automated analytical workflow was implemented that is much faster than traditional individual spectrum analysis. It allows the generation of structural data in quasi-real time. We describe initial results obtained from the automated high throughput (HT) data reduction and analysis of a sample library implemented through the 96 well-plate industrial standard. The results show that a fully automated HT-XAS technology based on existing industry standards is feasible and useful for the rapid elucidation of geometric and electronic structure of materials.
\end{abstract}

Keywords: High-throughput, XAS, XANES, EXAFS, catalysis.

PACS: $61.10 . \mathrm{Ht}, 81.16 . \mathrm{Hc}, 82.65 .-\mathrm{s}$.

\section{INTRODUCTION}

High throughput (HT) approaches can potentially increase research productivity considerably. HT methods developed originally in the context of pharmaceutical research [1] are currently extended to other research areas [2], and have become accepted in the catalysis community in recent years [3-4]. Detailed studies of catalytic materials using high-throughput spectroscopic techniques such as Infrared (IR) [5], Raman [6], X-ray Fluorescence (XRF) [7], Fluorescence Microscopy [8], Imaging Polarimetry [9] and Nuclear Magnetic Resonance (NMR) [10], have been reported. In catalysis it is crucial to establish novel routes that facilitate the discovery of new catalysts and the correlation of reaction outcomes with the atomic structure of the chemical species involved. Investigating the nature of catalytic precursors and their configuration with the support materials is the first step for determining catalyst stability, activity and selectivity in a particular chemical reaction [11-13]. X-ray absorption spectroscopy (XAS) is a synchrotron-based spectroscopic technique that provides this information by probing the local coordination geometry and electronic structure around X-ray-absorbing atoms [14]. It can investigate samples under in-situ reaction conditions and in any aggregation state and therefore is suitable for high-throughput experimentation.

We have developed XAS end-station instrumentation that allows the rapid elucidation of the metal local structure in a library of catalytic precursors based on a 96-wellplate sample array. This library contains 91 supported catalyst precursors in a ternary system $\mathrm{Cu} / \mathrm{Pt} / \mathrm{Au}$ prepared by impregnation of $\gamma-\mathrm{Al}_{2} \mathrm{O}_{3}$ with aqueous $\mathrm{CuCl}_{2}, \mathrm{PtCl}_{2}$ and $\mathrm{HAuCl}_{4}$ solutions. Here are presented the results from the automated HT data reduction and analysis of XAS spectra taken at the $\mathrm{Cu}$ K-edge. Results from the data analysis of spectra taken at $\mathrm{Au} \mathrm{L}_{\mathrm{III}}$ and $\mathrm{Pt} \mathrm{L}_{\mathrm{III}}$ will be extensively described in a forthcoming publication [15]. This HT spectral analysis was carried out using a set of scripts utilizing the IFEFFIT library, which provided values of coordination number, first shell distances and Debye-Waller factors

CP879, Synchrotron Radiation Instrumentation: Ninth International Conference, edited by Jae-Young Choi and Seungyu Rah

(C) 2007 American Institute of Physics 978-0-7354-0373-4/07/\$23.00 
for all samples. Phase changes were identified yielding valuable knowledge on the ternary catalyst precursors system.

A feasibility study on the inclusion of 2D correlation analysis of XAS spectra [16] as a feedback mechanism to the automated data acquisition system is also presented.

\section{EXPERIMENTAL}

\section{Library Preparation}

Catalyst precursors were prepared by wet impregnation of $\mathrm{CuCl}_{2}$ (Aldrich, 99.995\%), $\mathrm{PtCl}_{2}$ (Aldrich, 98\%) and $\mathrm{HAuCl}_{4}$ (Riedel-de Haën, 51\% Au) on $\gamma-\mathrm{Al}_{2} \mathrm{O}_{3}$ (D1011, BASF) support (200-450 $\mu$ m particle size). A standard 96 well plate was used to prepare and store the samples. The library comprises all possible combinations of $\mathrm{Cu}, \mathrm{Pt}$ and $\mathrm{Au}$ in the concentrations $0,0.1,1$ and $5 \mathrm{wt} \%$ leading to a total of 64 elements; 27 additional elements were added as permutations of $\mathrm{Cu}, \mathrm{Pt}$ and $\mathrm{Au}$ in concentrations of $0.1,1$ and $10 \mathrm{wt} \%$, resulting in a library of 91 elements. Each well containing the metal solution $(200 \mu \mathrm{l})$ was filled with $200 \mathrm{mg}$ of $\gamma-\mathrm{Al}_{2} \mathrm{O}_{3}$ corresponding to the pore volume of the support. The samples were then left to dry at room temperature. Figure la shows the Cu concentration in wt \% for each of the library members in the 96 well-plate.

\section{High-Throughput XAS Data Acquisition and Analysis}

XAS experiments were carried out at station 9.3 of the SRS in Daresbury Laboratory, UK [17]. Cu K-edge spectra were collected in fluorescence-yield mode using a Canberra 13 element Ge solid state detector. The EXAFS region was scanned in the k-scan mode from 2 to $14 \AA^{-1}$ using k-steps of $0.04 \AA^{-1}$. In the pre-edge region, data were recorded on an energy grid of $22 \mathrm{eV} / \mathrm{step}$ and with $1.0 \mathrm{eV} / \mathrm{step}$ in the XANES region. These parameters resulted in data acquisition times of 9 min per spectrum. An energy calibration of the beam was performed by collecting the transmission spectra of $\mathrm{Cu}$ reference foil at the $\mathrm{Cu}$ K-edge. An in-house built XYZ translation stage (Parker) was used for sample positioning. Data acquisition and automatic movement of the XYZ stage as well as synchronization with the station main computer through the TCP/IP protocol were achieved using a LabVIEW control system. The software allows the scan of a variety of library sizes, in several positions and angles with respect to the fluorescence detector.

Four individual spectra containing 5\% wt of $\mathrm{Au}$, $\mathrm{Pt}$ and/or $\mathrm{Cu}$ samples were initially analyzed and suitable starting parameters for background subtraction, normalization, k-weighting, Fourier transformation, edge-position, coordination number $(N)$, nearest neighbour distance $(R)$ and Debye-Waller factor $\left(\sigma^{2}\right)$ were determined. These values were then used as a priori information to the HT analysis software. This software is based on a series of Linux shell scripts which reads sequentially all the spectra in a subdirectory, applies the IFEFFIT background subtraction routine and performs first-shell fitting analysis according to a prescribed physical model. Theoretical amplitudes and phase functions used in the fitting procedure were calculated with FEFF6 [18]. All the fits were performed using multiple k-weightings of 1,2 and 3. The script reports the shift on the edge position, the edge-step (ES) values, the normalized data, the $\chi(\mathrm{k})$ data, the Fourier transformed data, the fitted curves and the resulting fitted values for $\mathrm{N}, \mathrm{R}$ and $\sigma^{2}$. This software routine performs single-shell EXAFS analysis on 96 samples at three different edges (i.e., a total of 288 spectra in approximately 20 minutes. In order to improve the versatility of the HT system, a feasibility study was carried out based on the inspection of $2 \mathrm{D}$ correlation maps from the collected spectra. These maps were built based on 2D XAS correlation analysis reported by Haider [16] and using the 2Dshige software [19].

\section{RESULTS AND DISCUSSION}

Some of the results from the HT-XAS analysis of spectra taken at the Cu K-edge are shown in Figs. $1 \mathrm{~b}$ and $1 \mathrm{c}$. These figures present a map of the $\mathrm{Cu}$ for one particular XAS parameter, namely the edge-step (Fig. 1b) and coordination number (Fig. 1c). The color black in the figures indicates either the absence of the metal under study or spectra with an extremely low S/N ratio. All results of the automated analysis were additionally cross-checked individually, and it was found that the information provided by the maps was reliable.

Most samples follow the expected strong correlation between concentration (Fig. 1a) and edge step (Fig. 1b), which supports the reliability of the HT analysis. In most cases, fits for the $\mathrm{Cu}$ K-edge are in line with the 4-fold 
(a)

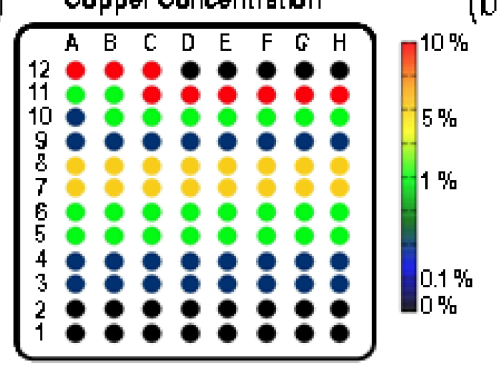

(b)

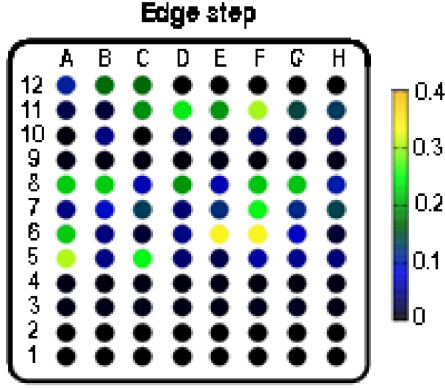

(c) Coordinetlon number

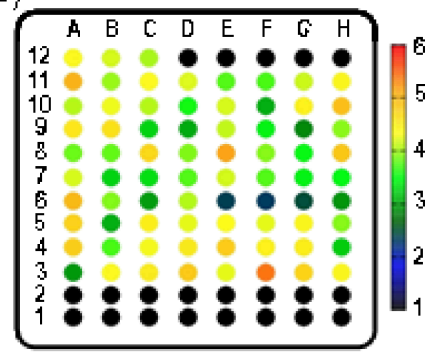

FIGURE 1. Color maps representing (a) the known copper loading (wt\%) of the catalysts, (b) the edge step values and (c) the coordination numbers found from the HT-XAS analysis.

coordination of $\mathrm{Cu}$ found in $\mathrm{CuCl}_{2}$ [20]. From immediate visual inspection one can observe high ES values (Fig. 1b) for samples E6 and $\mathrm{F} 6$ associated to $1 \mathrm{wt} \% \mathrm{Cu}$ metal. This may be due to an uneven distribution of $\mathrm{Cu}$ salt on the support or to irregularities in the synthesis procedure. In addition the $\mathrm{N}$ color map (Fig. 1c) clearly shows that E6, F6 and G6 present extremely low coordination numbers with non-physical significance. These samples were further analyzed individually leading to a metal local structure in line with the 12-fold coordination found in $\mathrm{Cu}$ metal. Figure 2a shows the XANES spectra of sample $\mathrm{A} 7$ corresponding to $\mathrm{CuCl}_{2}$ as expected from the impregnation process, sample E6 corresponding to reduced $\mathrm{Cu}$ structure and $\mathrm{Cu}$ foil shown for comparison purposes. The reduction is believed to take place at the expense of $\mathrm{Pt}(\mathrm{II})$, which oxidizes to $\mathrm{Pt}(\mathrm{IV})$, as indicated by an increase in the intense near-edge feature ("white line") in the Pt LIII XANES spectra [21]. This is illustrated in Fig. 2b, which compares spectra from samples E6 and E2; the latter contains only $\mathrm{PtCl}_{2}$ impregnated on alumina and therefore the XANES spectrum presents a reduced white line feature. Figure $2 b$ also shows the Pt foil XANES spectrum for comparison.

The selected results described above demonstrate that HT XAS analysis is an efficient tool for rapid structural phase identifications and may be used in the future for the determination of structural changes under reaction conditions.

Figure 3 shows the 2D XAS synchronous correlation maps for the normalized spectra under discussion. Figure 3a corresponds to the synchronous correlation spectra of samples A7 and E6. One can identify peaks corresponding to spectral variations between the two $\mathrm{Cu} \mathrm{K}$-edge spectra. This is in line with the difference in spectral features associated to different local structures i.e. $\mathrm{Cu}$ metal and $\mathrm{CuCl}_{2}$. Figure $3 \mathrm{~b}$ shows the 2D XAS synchronous correlation spectra for samples E6, F6 and G6. In contrast to Fig. 3a, spectral correlations are found that appear as a blurry image with "crossing lines" that are associated with noise in the data [22]. This sole presence of noise in this figure is expected, as all three samples presented the same 12-fold $\mathrm{Cu}$ metal structure. Finally Fig. $3 \mathrm{c}$ shows the correlation map of two identical spectra (both from sample A7) as an illustration of total correlation. These results show that using 2D XAS correlation analysis as a feedback mechanism may, with minimum effort, (i) improve the rapid data acquisition by discriminating between spectral noise levels and (ii) provide a strategy for the rapid determination of possible phase changes under reaction conditions.

(a)

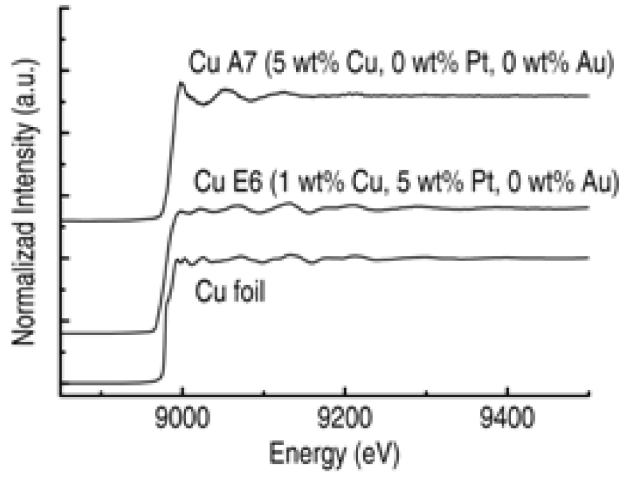

(b)

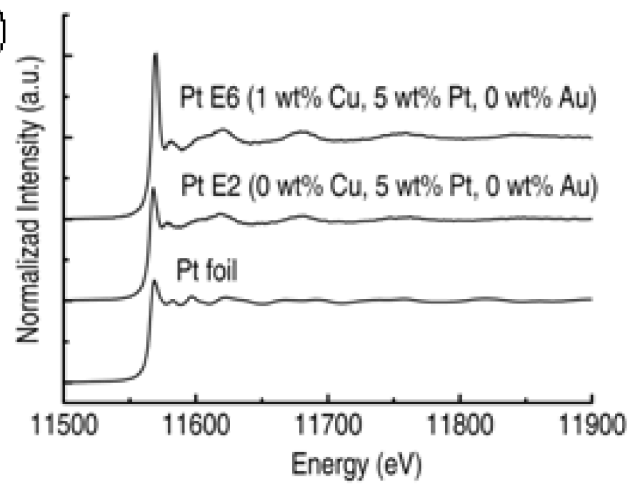

FIGURE 2. Normalized XANES spectra of selected samples taken at the (a) Cu K-edge and (b) $\mathrm{Pt} \mathrm{L}_{\mathrm{III}}$-edge. 
(a)

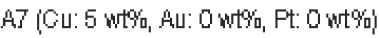

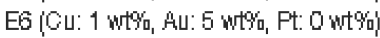

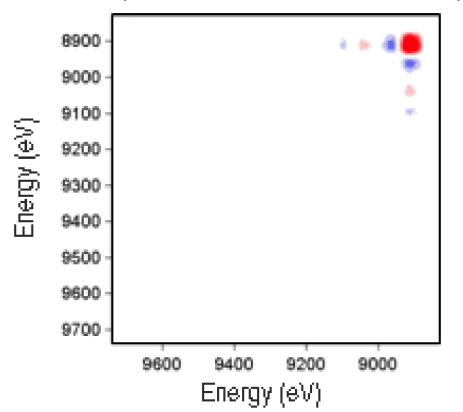

(b) E6 [Cu: $1 w+\%$, Au: $5 w$ t\%, Ft: 0wt\%) F6 (Cu: $1 w+\%, A u: 5 w+\%_{0}$, Ft: $\left.0.1 w+\%\right)$

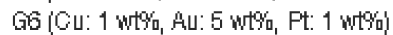

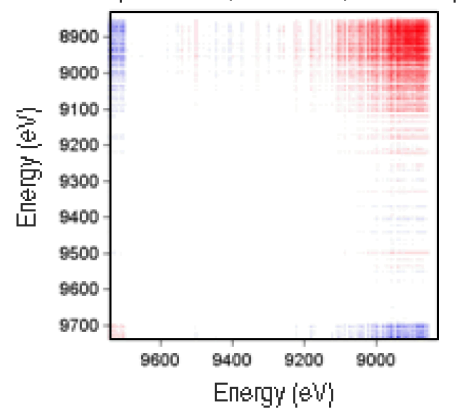

(c) A] (Cu: $5 w \%_{0}$, Au: $0 w \%$, Ft: 0 $\left.w+\%\right)$ A7 (Cu: $5 w t \%_{0}$, Au: $0 w t \%_{0}$, Ft: $0 w t \%$ )

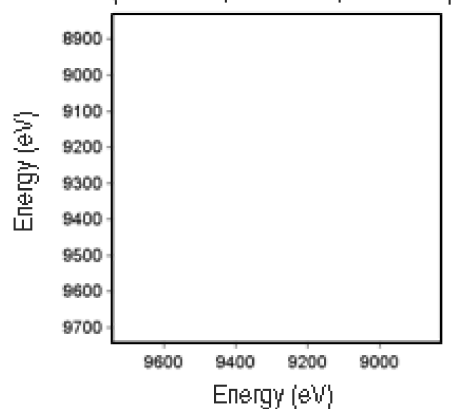

FIGURE 3. 2D XAS correlation spectra taken at the CuK-edge for samples: (a) A7 and E6, (b) E6, F6 and G6 and (c) A7.

\section{CONCLUSIONS}

We have demonstrated that HT-XAS can significantly reduce the amount of time needed to acquire and analyze XAS data as compared to standard XAS experimentation, yielding rapid information about correlations between structural parameters and providing fast and reliable structural phase identification.

\section{ACKNOWLEDGMENTS}

This work was supported through EPSRC research grants GR/S85801/01 and GR/S85818/01. The CCLRC Synchrotron Radiation Source (SRS) at Daresbury Laboratory is acknowledged for providing beamtime at station 9.3 under award number 44135 .

\section{REFERENCES}

1. R. A. Houghten, C. Pinilla, S. E. Blondelle, J. R. Appel, C. T. Dooley and C. T. Cuervo, Nature 354, 84-86 (1991).

2. R. J. Hendershot, C. M. Snively and J. Lauterbach, Chem. Eur. J. 11, 806-814 (2005).

3. T. Zech, G. Bohner, O. Laus, J. Klein and M. Fischer, Rev. Sci. Instrum 76, 62215-1-8 (2005).

4. C. Klanner, D. Farrusseng, L. Baumes, C. Mirodatos, F. Schuth, QSAR. Comb. Sci. 22, 729-735 (2003).

5. A. Venimadhav, K. A. Yates, M. G. Blamire, J. Comb. Chem. 7, 85-89 (2005).

6. A. Leugers, D. R. Neithamer, L. S. Sun, J. E. Hetzner, S. Hilty, S. Hong, M. Krause, K. Beyerlein, J. Comb. Chem. 5, 238$244(2003)$.

7. T. C. Miller, G. Mann, G. J. Havrilla, C. A. Wells, B. P. Warner, R. T. Baker, J. Comb. Chem. 5, 245-252 (2003).

8. M. M. Taniguchi, R. A. Farrer, J. Fourkas, J. Comb. Chem. 7, 54-57 (2005).

9. P. R. Gibbs, C. S. Uehara, P. T. Nguyen, R. C. Willson, Biotechnol. Prog. 19, 1329-1334 (2003).

10. R. A. Kautz, W. K. Goetzinger, B. L. Karger, J. Comb. Chem. 7, 14-20 (2005).

11. C. L. Bianchi, P. Canton, N. Dimitratos,F. Porta, L. Patri, Catalysis Today 102-103, 203-212 (2005).

12. J. Sloczyński, R. Grabowski, A. Kozlowska, P. Olszewski, J. Stoch, J. Skrzypek, M. Lachowska, Appl. Catal. A 278, 11-23 (2004).

13. M.Ritcher, M. Langpape, S. Kolf, G. Grubert; R. Eckelt, J. Radnik, M. Schneider, M. -M. Pohl, R. Fricke, Appl. Catal. B 36, 261-277 (2002).

14. J. J. Rehr, A. L. Ankudinov, S. I. Zabinsky, Catal. Today 39, 263-269 (1998).

15. N. Tsapatsaris, A. Beesley, N. Weiher, M. Tromp, J. Evans, A. Dent, I. Harvey, S. Hayama, S. L.M. Schroeder to be submitted to J. Comb. Chem.

16. P. Haider, Y. Chen, S. Lim, G. L. Haller, L. Pfefferle and D. Ciuparu, J. Am. Chem. Soc. 127, 1906-1912, (2005).

17. Dent, A. J.; Derst, G.; Van Dorssen, G. E.; Mosselmans, J. F. M. Daresbury Synchrotron Radiation Source (SRS), Manual Station 9.3. http://www.srs.dl.ac.uk/srs/stations/station9.3.htm.

18. S. I. Zabinsky, J. J. Rehr, A. Ankudinov, R. C. Albers, M. Eller, Phys. Rev. B 52, 2995-3009 (1995).

19. 2Dshige (c) Shigeaki Morita, Kwansei-Gakuin University, 2004-2005.

20. P. C. Burns, F. C. Hawthorne, Am. Mineral. 78, 187-189 (1993).

21. R. Ayala, M. E. Sanchez, S. Diaz-Moreno, V. A. Sole, A. Munoz-Paez, J. Phys. Chem. B. 105, 7588-7593 (2001).

22. M. A. Czarnecki, Appl. Spectrosc. 52, 1583-1590 (1998). 